\title{
What Causes the High Apparent Speeds in Chromospheric and Transition Region Spicules on the Sun?
}

\author{
Bart De Pontieu $^{1,2}$ (D) , Juan Martínez-Sykora ${ }^{1,3}$ (D), and Georgios Chintzoglou ${ }^{1,4}$ (D) \\ ${ }^{1}$ Lockheed Martin Solar and Astrophysics Laboratory, Palo Alto, CA 94304, USA; bdp@lmsal.com \\ ${ }^{2}$ Institute of Theoretical Astrophysics, University of Oslo, P.O. Box 1029 Blindern, NO-0315 Oslo, Norway \\ ${ }^{3}$ Bay Area Environmental Research Institute, Petaluma, CA 94952, USA \\ ${ }^{4}$ University Corporation for Atmospheric Research, Boulder, CO 80307-3000, USA \\ Received 2017 June 22; revised 2017 October 5; accepted 2017 October 9; published 2017 October 23
}

\begin{abstract}
Spicules are the most ubuiquitous type of jets in the solar atmosphere. The advent of high-resolution imaging and spectroscopy from the Interface Region Imaging Spectrograph (IRIS) and ground-based observatories has revealed the presence of very high apparent motions of order $100-300 \mathrm{~km} \mathrm{~s}^{-1}$ in spicules, as measured in the plane of the sky. However, line of sight measurements of such high speeds have been difficult to obtain, with values deduced from Doppler shifts in spectral lines typically of order $30-70 \mathrm{~km} \mathrm{~s}^{-1}$. In this work, we resolve this long-standing discrepancy using recent $2.5 \mathrm{D}$ radiative MHD simulations. This simulation has revealed a novel driving mechanism for spicules in which ambipolar diffusion resulting from ion-neutral interactions plays a key role. In our simulation, we often see that the upward propagation of magnetic waves and electrical currents from the low chromosphere into already existing spicules can lead to rapid heating when the currents are rapidly dissipated by ambipolar diffusion. The combination of rapid heating and the propagation of these currents at Alfvénic speeds in excess of $100 \mathrm{~km} \mathrm{~s}^{-1}$ leads to the very rapid apparent motions, and often wholesale appearance, of spicules at chromospheric and transition region temperatures. In our simulation, the observed fast apparent motions in such jets are actually a signature of a heating front, and much higher than the mass flows, which are of order $30-70 \mathrm{~km} \mathrm{~s}^{-1}$. Our results can explain the behavior of transition region "network jets" and the very high apparent speeds reported for some chromospheric spicules.
\end{abstract}

Key words: magnetohydrodynamics (MHD) - methods: numerical - radiative transfer - Sun: atmosphere - Sun: chromosphere - Sun: transition region

Supporting material: animations

\section{Introduction}

Jets are very common in the solar chromosphere. They are most often detected as rapidly evolving linear features in which plasma appears to be accelerated and sometimes heated, with the bulk of the plasma flow often penetrating into the overlying and much hotter corona. There is a wide variety of chromospheric jets, including surges, penumbral microjets, anemone jets, macrospicules, and spicules (Raouafi et al. 2016). To fully understand the impact of these different jets on the mass and energy balance of the low solar atmosphere, it is key to better understand the mechanisms that drive these jets. In this Letter, we focus on spicules, the most common of the solar jets, as they have the largest potential to impact the overlying corona or solar wind (Beckers 1968; Athay \& Holzer 1982; De Pontieu et al. 2011; McIntosh et al. 2011). During the past decade, significant progress has been made to better understand spicules and how they impact the solar atmosphere, but key questions remain.

At least two different types of spicules have been reported. The so-called type I spicules, identified as dynamic fibrils or mottles on the disk, show both apparent motions and mass flows of maximally $10-40 \mathrm{~km} \mathrm{~s}^{-1}$ and appear to be driven by magneto-acoustic shocks that form when convective motions, p-modes or magnetic disturbances propagate upward into the chromosphere (Hansteen et al. 2006; De Pontieu et al. 2007a; Rouppe van der Voort et al. 2007; Martínez-Sykora et al. 2009). The transition region response to type I spicules appears to be limited to brightenings at the top of the spicule
(Skogsrud et al. 2016). The bulk of spicules observed at the solar limb are, however, type II spicules. These are much faster, showing apparent motions with maximum speeds of $30-150 \mathrm{~km} \mathrm{~s}^{-1}$ (De Pontieu et al. 2007b; Pereira et al. 2012). Such speeds dominate the spicules seen in the chromospheric $\mathrm{Ca}$ II $\mathrm{H}$ passband, which recent results indicate are the initial upward phase of longer-lived spicules that show up and downward motion when viewed over a wide range of chromospheric and TR passbands (Skogsrud et al. 2015). In contrast to type I spicules, type II spicules often appear to be associated with heating to at least transition region temperatures (De Pontieu et al. 2014a; Rouppe van der Voort et al. 2015). Their short-lived ( 20-50 s) transition region counterparts often appear in Si IV and C II slit-jaw images (SJI) from the Interface Region Imaging Spectrograph (IRIS; De Pontieu et al. 2014b) as linear features that emanate from magnetic network with even higher apparent speeds of $80-300 \mathrm{~km} \mathrm{~s}^{-1}$ (Tian et al. 2014; Narang et al. 2016). The transition region counterparts appear to be shorter and slower in quiet Sun than in coronal holes, presumably because of differing magnetic field configurations (Narang et al. 2016). Such high apparent speeds have also been found in other upperchromospheric lines such as Ly $\alpha$ (Kubo et al. 2016). There is, however, a large discrepancy between these high apparent speeds and the actual mass flows in spicules as deduced from Doppler shift measurements. For example, the on-disk chromospheric counterparts of type II spicules are rapid blueshifted events which show typical Doppler shifts of $20-50 \mathrm{~km} \mathrm{~s}^{-1}$ (Sekse et al. 2012, 2013). Similarly, Doppler 

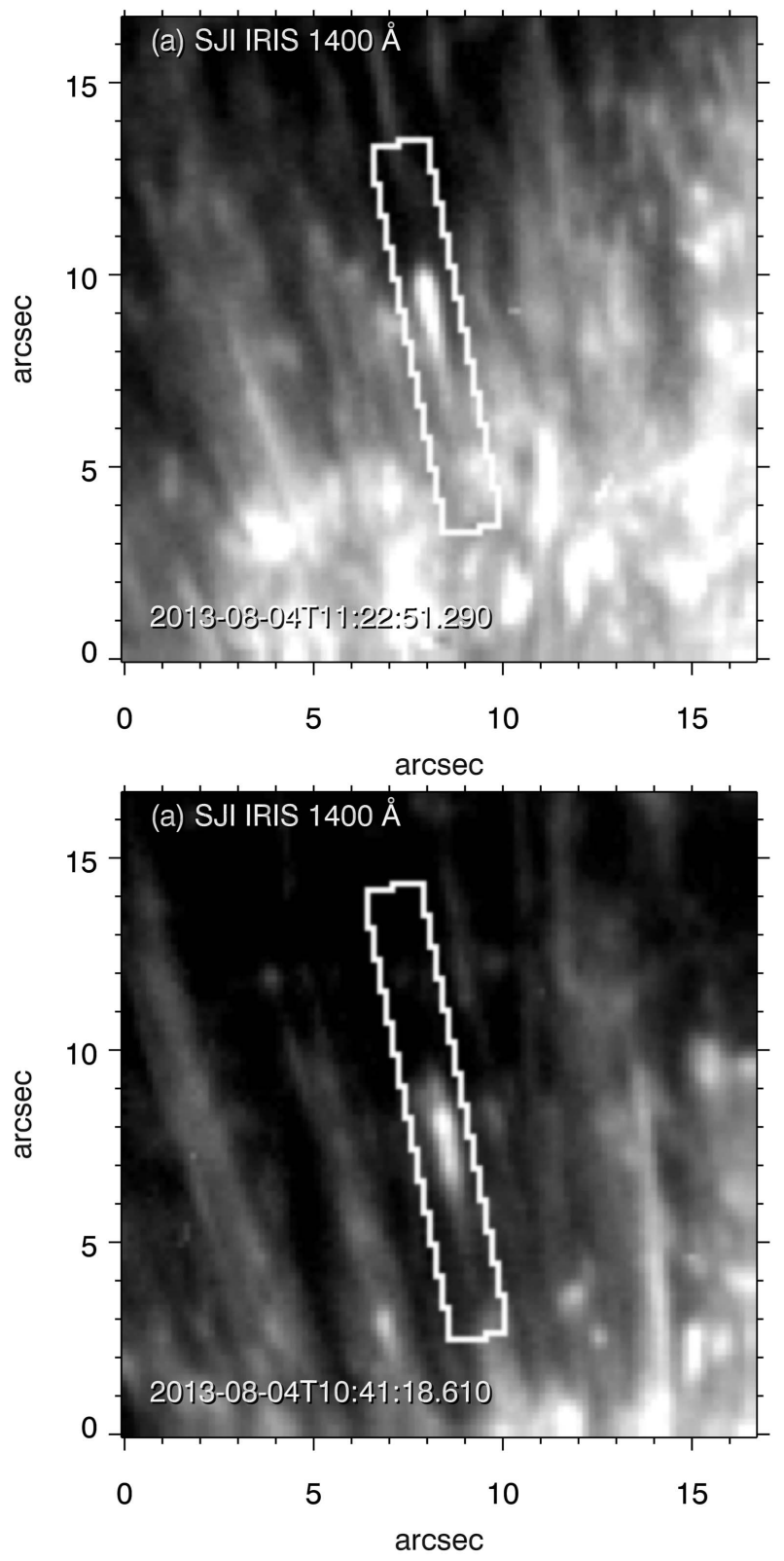
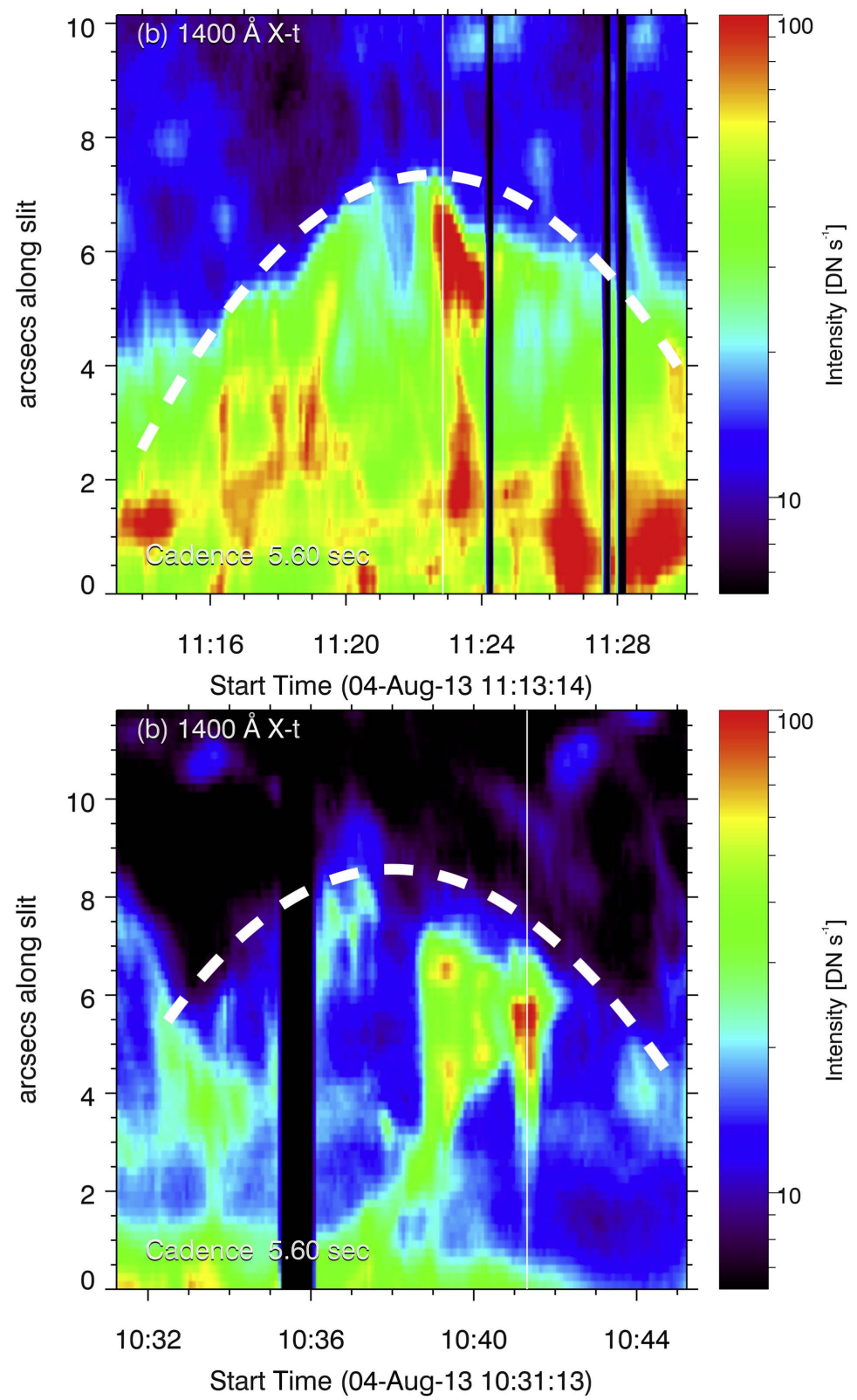

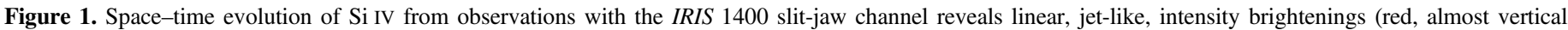

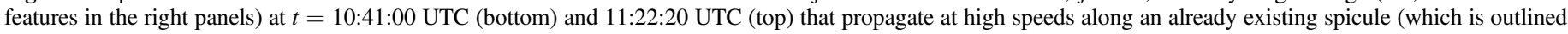

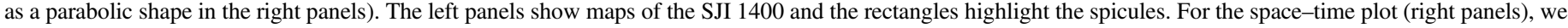

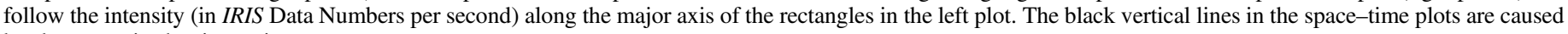
by data gaps in the timeseries.

(Animations (a) and (b) of this figure are available.)

shift measurements in transition region lines of spicules show velocities up to only $50-70 \mathrm{~km} \mathrm{~s}^{-1}$ (Rouppe van der Voort et al. 2015).

The viewing geometry could in principle explain some of the differences between the apparent motions, often measured at the limb, and Doppler shifts, typically measured on the disk. However, the fact is that Doppler shifts of $100-300 \mathrm{~km} \mathrm{~s}^{-1}$ are extremely uncommon on the disk and they would be expected if the plane-of-the-sky motions were real mass motions. More importantly, the IRIS observations of the apparent and real mass motions of transition region counterparts of spicules (Tian et al. 2014; Rouppe van der Voort et al. 2015) are both on the disk, i.e., with similar viewing angles. These differences thus appear to be real and not easy to explain, thus providing strict constraints for any theoretical model of spicules. A multitude of theoretical models have been developed in the past, but until recently no single model has been able to simultaneously explain in detail the ubiquity, dynamic, and thermal evolution and visibility and appearance in various chromospheric and transition region observables (for a review, see Sterling 2000; Tsiropoula et al. 2012). Here, we exploit a recent numerical model based on 2.5D radiative MHD simulations that proposes that type II spicules occur when magnetic tension, created through the interaction between strong flux concentrations and weaker, granular-scale, magnetic flux concentrations, can diffuse into the chromosphere through ambipolar diffusion. In the chromosphere, the sudden release of tension drives strong flows and ion-neutral interactions lead to heating of the spicular 


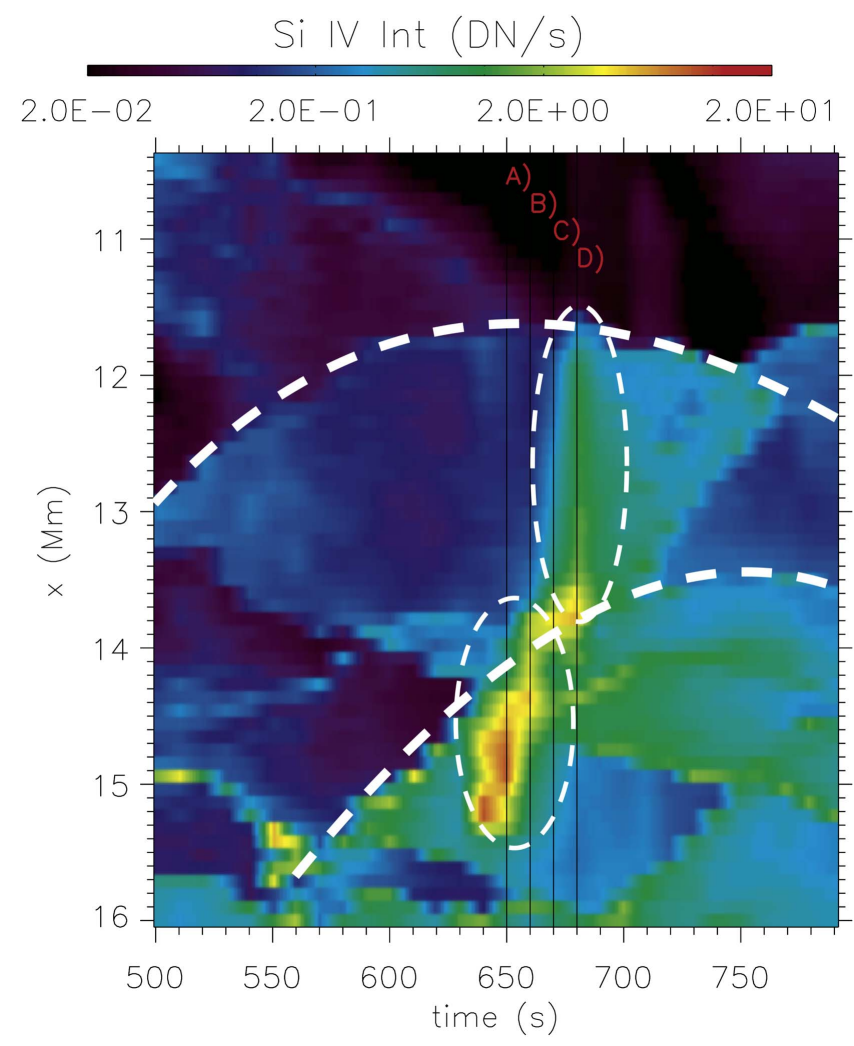

Figure 2. Space-time evolution of a top view of Si IV (in IRIS Data Numbers per second assuming the signal would cover an IRIS pixel of 0 ." $166 \times 0$." 166) from a $2.5 \mathrm{D}$ radiative MHD simulation reveals two jet-like features (inside the dashed white ovals) that propagate at high apparent speeds at $t=630 \mathrm{~s}$ $(x=14-15.5 \mathrm{Mm})$ and at $t=680 \mathrm{~s}(x=11.5-13.5 \mathrm{Mm})$, with similar speeds as seen in the observations (Figure 1). These jets form on already existing spicules (whose space-time path is outlined with dashed white parabolas). The vertical solid lines are the instances shown in Figures 3 and 4. This plot is based on a top view of the simulation shown in Figure 3, i.e., the $x$-coordinates are the same in both figures.

plasma (Martínez-Sykora et al. 2017a). We compare the model predictions with observations from IRIS.

\section{Observations}

We use IRIS $1400 \AA$ SJI of an on-disk plage region (NOAA AR 11809) centered at $[x, y]=[383,124]^{\prime \prime}$, with a field of view of $119^{\prime \prime} \times 120^{\prime \prime}$. The observations were taken on 2013 August 4 10:38-11:41 UTC. IRIS was in sit-and-stare mode (IRIS OBS-ID 4043007648) taking only the $1400 \AA$ SJI passband images with cadence of $5.6 \mathrm{~s}$ and exposure time of $4 \mathrm{~s}$. We use level-2 IRIS data that was corrected for flat-field, dark current, geometry, and co-alignment (De Pontieu et al. 2014b).

\section{Simulations}

We compare our observations with a numerical simulation that uses Bifrost, a radiative MHD code (Gudiksen et al. 2011). This code solves the MHD equations including radiative transfer from the photosphere to corona (Hayek et al. 2010; Carlsson \& Leenaarts 2012) and thermal conduction along the magnetic field. We also include ion-neutral interaction effects by using the Generalized Ohm's law, i.e., by adding the ambipolar diffusion and Hall term to the induction equation (Martínez-Sykora et al. 2012, 2017b).
The simulation analyzed here is the $2.5 \mathrm{D}$ radiative MHD model described in detail in Martínez-Sykora et al. (2017a, 2017b). The numerical domain ranges from the upper layers of the convection zone ( $3 \mathrm{Mm}$ below the photosphere) to the self-consistently maintained hot corona ( $40 \mathrm{Mm}$ above the photosphere) and $90 \mathrm{Mm}$ along the horizontal axis. We use this particular model because it is the first Bifrost simulation in which type II spicules occur ubiquitously. Martínez-Sykora et al. (2017a) show that ambipolar diffusion plays a dominant role in the formation of spicules. To compare our simulation with observations, we calculate the synthetic Si IV intensity assuming equilibrium ionization and the optically thin approximation, similar to the methods used by Hansteen et al. (2010).

\section{Results}

Measurements of apparent motions of spicules in the plane of the sky reveal a wide range of velocities, with the highest velocities recorded in IRIS SJI. These are sensitive to emission from lower transition region lines like C II $1335 \AA$ (SJI $1330 \AA$ ) and Si IV $1402 \AA$ (SJI $1400 \AA$ ). Previous results indicate that velocities of $80-250 \mathrm{~km} \mathrm{~s}^{-1}$ (Tian et al. 2014) are common. A statistical study shows that apparent velocities of up to $350 \mathrm{~km} \mathrm{~s}^{-1}$ are sometimes seen, with clear differences between coronal hole $\left(190 \pm 60 \mathrm{~km} \mathrm{~s}^{-1}\right)$ and quiet Sun $\left(110 \pm 40 \mathrm{~km} \mathrm{~s}^{-1}\right)$ spicules (Narang et al. 2016). Such fast motions in spicules also occur in active regions. Figure 1 shows two examples of spicules (left panels) that emanate from strong magnetic flux concentrations in plage as observed in the IRIS SJI $1400 \AA$ A channel. The space-time evolution (right panels) of these two different spicules show similar behavior. We see very rapid (i.e., almost vertical in the space-time plot) linear brightenings (red in the right panels) that shoot outward from the spicule footpoints. These fast apparent jets appear to occur along an existing spicule and cover the full length of the spicule $\left(\sim 4^{\prime \prime}\right.$, i.e., $\left.3 \mathrm{Mm}\right)$ within a few tens of seconds. The previously existing spicule is seen as a very faint (note the logarithmic intensity scale in the right panels) parabolic path with a lifetime of $\sim 10$ (top) and 8 (bottom) minutes. Most spicules do not show such clear parabolic profiles in space-time plots derived from IRIS slitjaws. In those cases, the only observed features are the rapid apparent jets. This is especially the case in quiet Sun and coronal hole spicule observations. The fast apparent jets are sometimes repetitive, with linear brightenings recurring along the same spicules (the bottom panel of Figure 1).

Similar behavior is seen in the synthetic transition region observables calculated from our numerical simulation. Upper chromospheric and transition region observables reveal the presence of linear features with very fast apparent motions associated with the simulated spicules. The example in the space-time plot of synthetic Si IV $1402 \AA$ intensity in Figure 2 reveals two very fast apparent jets that appear almost consecutively, one next to the other. These apparent jets occur on an already fully evolved spicule which is "filled" along its whole length with a strong Si IV $1402 \AA$ brightening within 10 seconds. The first event is at $x=[13.9,15.4] \mathrm{Mm}$, and $t \sim 650 \mathrm{~s}$ and the second event is at $x=[11.2,13.6] \mathrm{Mm}$, and $t \sim 680 \mathrm{~s}$. When seen from above (top view along the negative $z$-axis), these linear features would show speeds of roughly $150-200 \mathrm{~km} \mathrm{~s}^{-1}$. When viewed from a line of sight that is perpendicular to the simulated spicules, the apparent motions would be even faster (200-300 $\left.\mathrm{km} \mathrm{s}^{-1}\right)$. Our simulations show that the apparent jets (observed in TR lines) occur around the time of the maximum extent of the chromospheric 

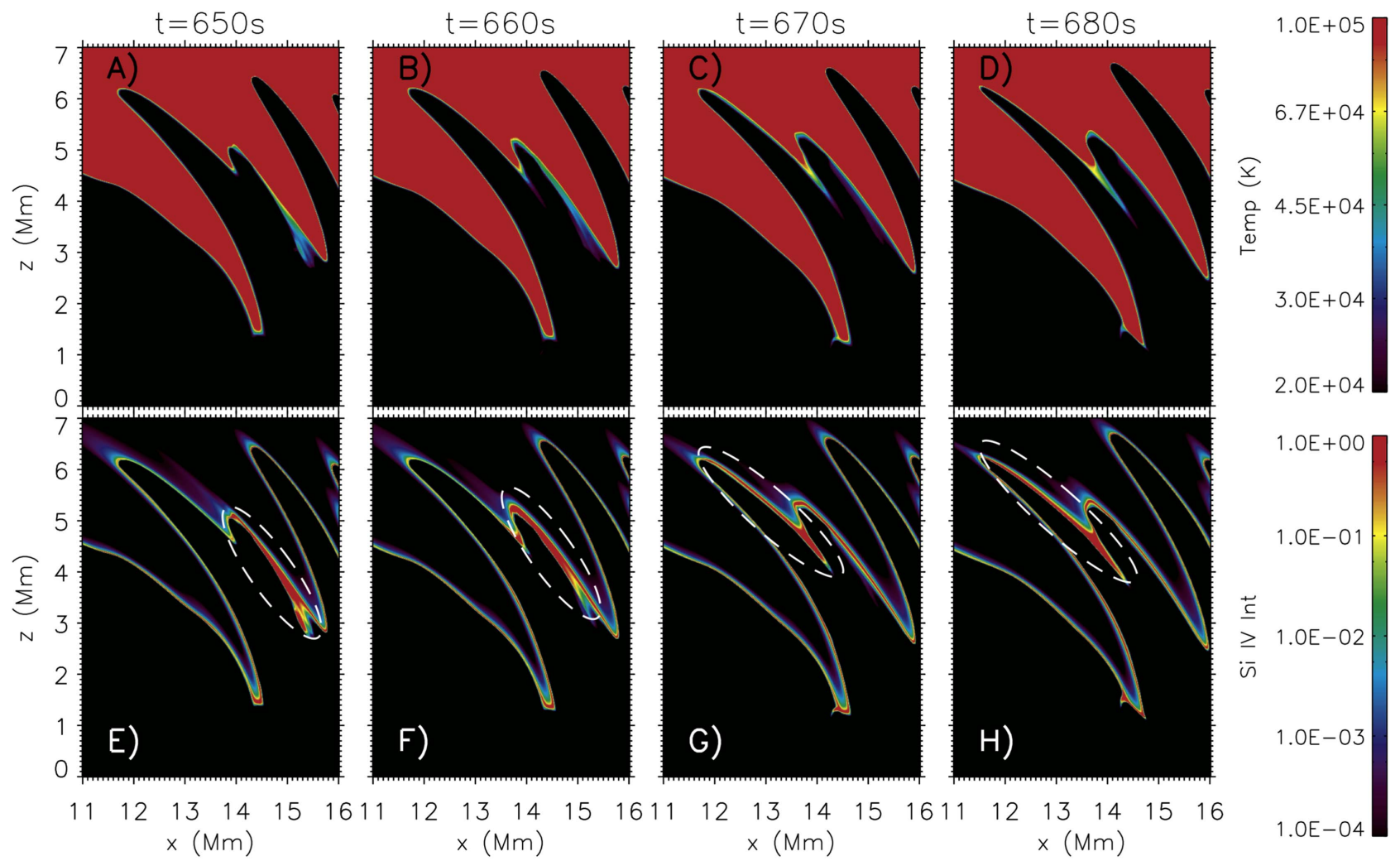

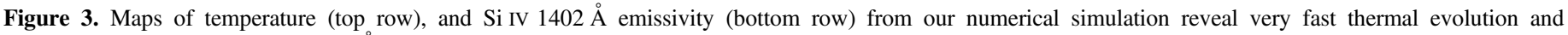

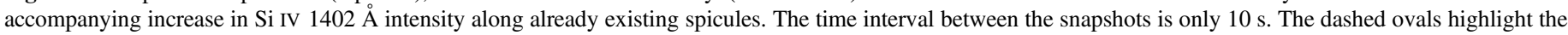

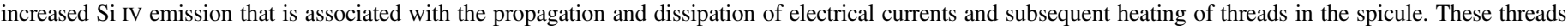

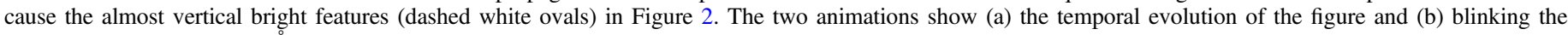
temperature and Si IV 1402 A emissivity plots.

(Animations (a) and (b) of this figure are available.)

spicule (visible as parabolic paths overdrawn in Figure 2), i.e., when the chromospheric mass flows are already much reduced (of order $<20 \mathrm{~km} \mathrm{~s}^{-1}$ ) compared to the very high chromospheric mass flows (up to $70 \mathrm{~km} \mathrm{~s}^{-1}$ ) during the initial acceleration of the jet. At the time of the occurrence of the TR counterparts, their apparent speeds are thus much higher than the chromospheric mass flows.

The cause for these apparent Si IV $1402 \AA$ jets is elucidated by Figure 3 which shows the temporal evolution of the temperature and the synthetic Si IV $1402 \AA$ intensity. In the center of the domain $(x=12-14.5 \mathrm{Mm})$, we can see two fully developed spicules that undergo very rapid thermal changes. The spicule located on the left-hand side $(x \sim 12-14 \mathrm{Mm})$ shows an increase of Si IV intensity from its footpoint toward the top, all along the right side during the time range $t=660-680 \mathrm{~s}$. This sudden increase in transition region spectral line intensity is caused by a thermal perturbation that propagates upward along the spicule and travels roughly $3 \mathrm{Mm}$ in about 10 to $20 \mathrm{~s}$, i.e., with a propagation speed of $150-300 \mathrm{~km} \mathrm{~s}^{-1}$ along the spicule (Figure 3). This heating front is localized in a very narrow region within the spicule and leads to a significant increase in the Si IV $1402 \AA$ intensity. The propagation speed of the heating front is much faster than the actual mass flows at this time. The spicule on the right-hand side of Figure 3 similarly shows a rapidly propagating heating front that causes the earlier apparent jet that is visible at $x=14-16 \mathrm{Mm}$ in Figure 2.
In our simulation, the appearance of these linear features with high apparent propagation speeds is caused by the following mechanism. After the simulated type II spicules are already fully formed, the low-density spicular environment causes the collision frequency between ions and neutrals to drop, resulting in strong ambipolar diffusion along the spicules. Meanwhile, in the photosphere below the spicule, magnetic energy is built up due to the convective motions. Large currents in the upper photosphere are seen in red in the bottom row of Figure 4. Eventually, these currents escape into the chromosphere. For instance, see the current on right-hand side of the left spicule between $t=[660,680] \mathrm{s}$. These electric currents propagate along the magnetic field at Alfvénic speeds, driven by tension and/or transverse waves. The map of Alfvén speeds shown in the top panel of Figure 5 reveals values between 150 and $450 \mathrm{~km} \mathrm{~s}^{-1}$ within the spicule. Because the ambipolar diffusion is so high along the spicule, the magnetic energy is dissipated on very short timescales (a few seconds). The heating from ambipolar diffusion is shown in the top row of Figure 4. This strong heating increases plasma temperatures from chromospheric $\left(\sim 8 \times 10^{3} \mathrm{~K}\right)$ to upper-chromospheric or transition region temperatures $\left(10^{4}-10^{5} \mathrm{~K}\right)$ on short timescales, i.e., between $t=660$ and $680 \mathrm{~s}$ for the left spicule. Similar to the intensity and temperature maps shown in Figure 3, the heating and current are very collimated and localized in a very narrow region along the spicule $(\sim 100 \mathrm{~km})$. The cause for the high apparent speeds is illustrated further in the bottom row of Figure 5, which shows a space-time plot along the length of 
$t=650 s$
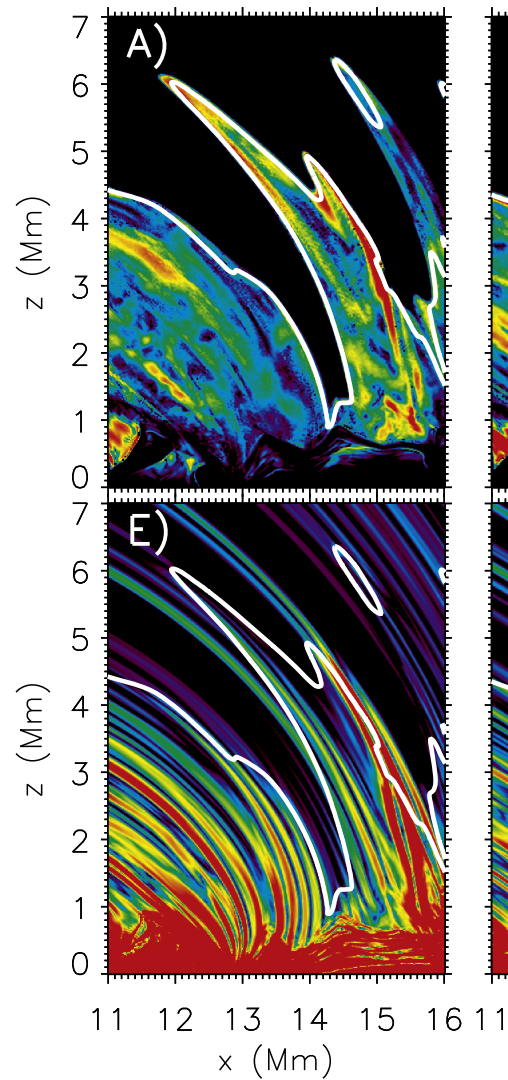

$t=660 \mathrm{~s}$
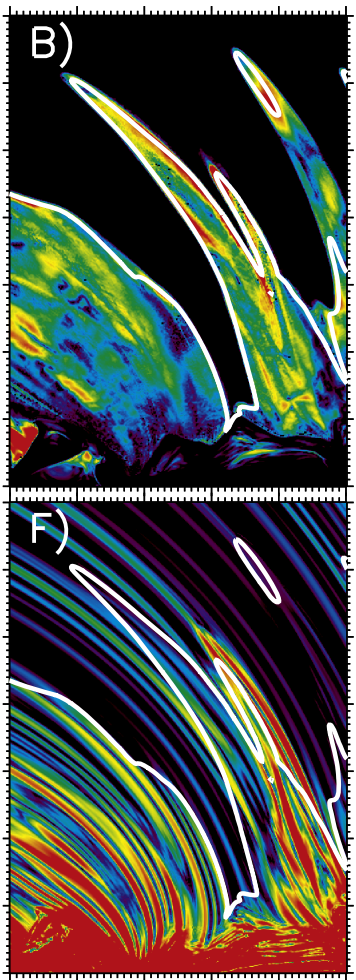

$\begin{array}{lllll}12 & 13 & 14 & 15 & 161\end{array}$ $\times(\mathrm{Mm})$
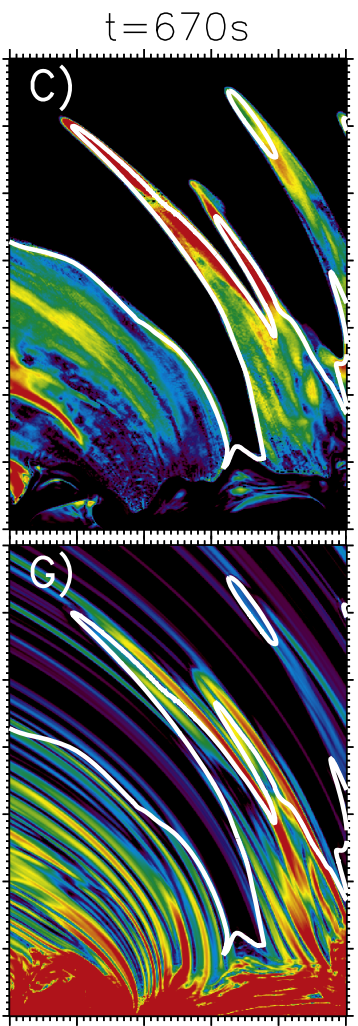

$1213 \quad 14 \quad 15 \quad 1611$ $\times(\mathrm{Mm})$
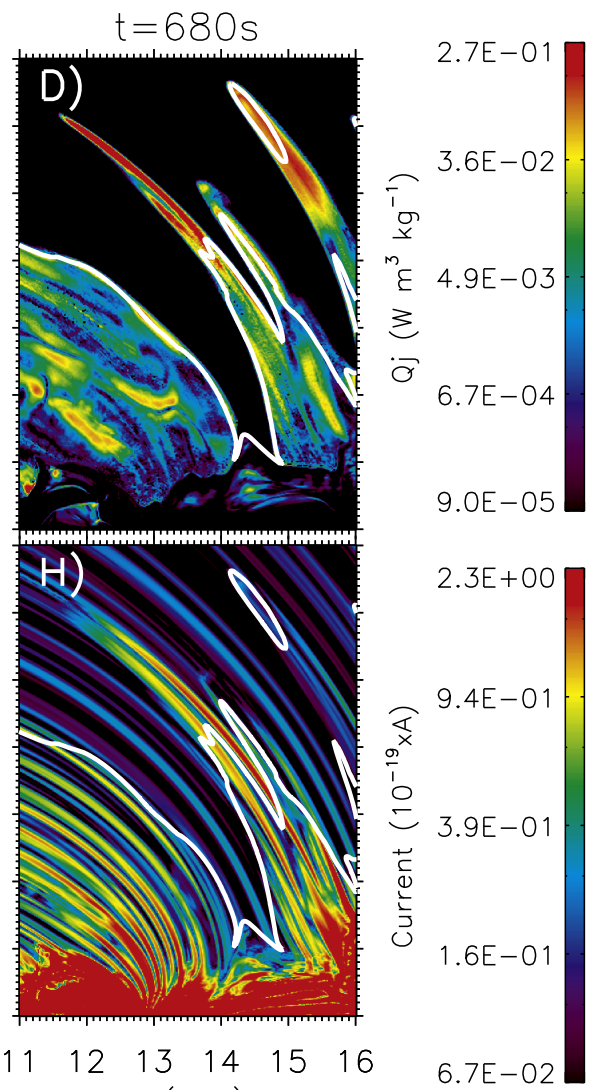

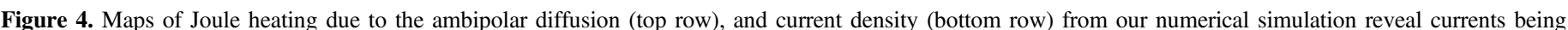

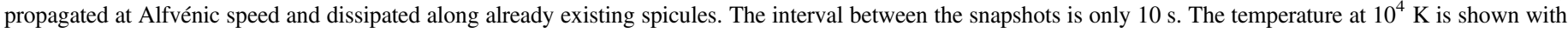
white contours.

(and summed across) the two spicules. The first apparent jet occurs in the right spicule $(t=630 \mathrm{~s})$ and is associated with a strong current and associated heating front that causes a large increase in Si IV $1402 \AA$ intensity. The second apparent jet follows a similar scenario and occurs at $t=670 \mathrm{~s}$ in the left spicule at somewhat greater heights. These timescales, speeds, and synthetic observables all are in agreement with the observations (Tian et al. 2014; Narang et al. 2016). We note that this is not the only possible cause for fast apparent jets at transition region temperatures. As mentioned, the initial violent acceleration of the spicule often involves mass motions of order $70 \mathrm{~km} \mathrm{~s}^{-1}$; thus, it is possible that the low-end of the reported velocity range is associated with real mass motions. However, our results suggest that the bulk of the very high apparent speeds are likely caused by heating fronts that propagate at Alfvénic speeds.

\section{Discussion and Conclusions}

Our results suggest that fast apparent motions seen in linear features that appear to be the transition region counterparts of spicules (Tian et al. 2014) are often not caused by real mass motions. Instead, these motions in the plane of the sky are of order $100-300 \mathrm{~km} \mathrm{~s}^{-1}$ and may be caused by the rapid propagation, at Alfvénic speeds, of heating fronts. A detailed comparison with our $2.5 \mathrm{D}$ radiative MHD simulation indicates that these heating fronts are an integral part of the spicule formation process and caused by the rapid dissipation of electric currents that propagate from the photosphere through the spicule into the corona. The dissipation mechanism is driven by the collisions between ions and neutrals: in the lowdensity spicular environment, the ion-neutral collision frequency is significantly decreased leading to slippage between the ionized and neutral particles. This slippage causes dissipation of currents and subsequent heating of plasma.

Our simulation can explain the well-known mismatch between the speeds measured from imaging timeseries $\left(100-300 \mathrm{~km} \mathrm{~s}^{-1}\right)$ and spectroscopic measurements of Doppler shifts $\left(<100 \mathrm{~km} \mathrm{~s}^{-1}\right)$. It also suggests that the linear shape of such features, often taken as a signature for the presence of a jet, may be misleading. In our case, the linear feature forms because plasma is heated along an already formed jet, but the rapid formation of the linear features is not necessarily associated with the formation of the jet. This means that the observed motions are not caused by mass motions, which impacts the estimated mass and kinetic energy flux that these jets may provide into the solar wind (Tian et al. 2014). Our results not only provide an explanation for the rapid "network jets" observed with IRIS (Tian et al. 2014), but could also provide an explanation for the rapid upper-chromospheric signals that have been recently observed with the CLASP rocket instrument (Kubo et al. 2016).

The excellent match between the simulation and observations provides more support for the spicule formation mechanism proposed by Martínez-Sykora et al. (2017a). This model proposes that spicules form when ambipolar diffusion in the low chromosphere allows magnetic fields, resulting from the interaction between strong network or plage fields and the ubiquitous, weak, granular-scale magnetic fields, to emerge 

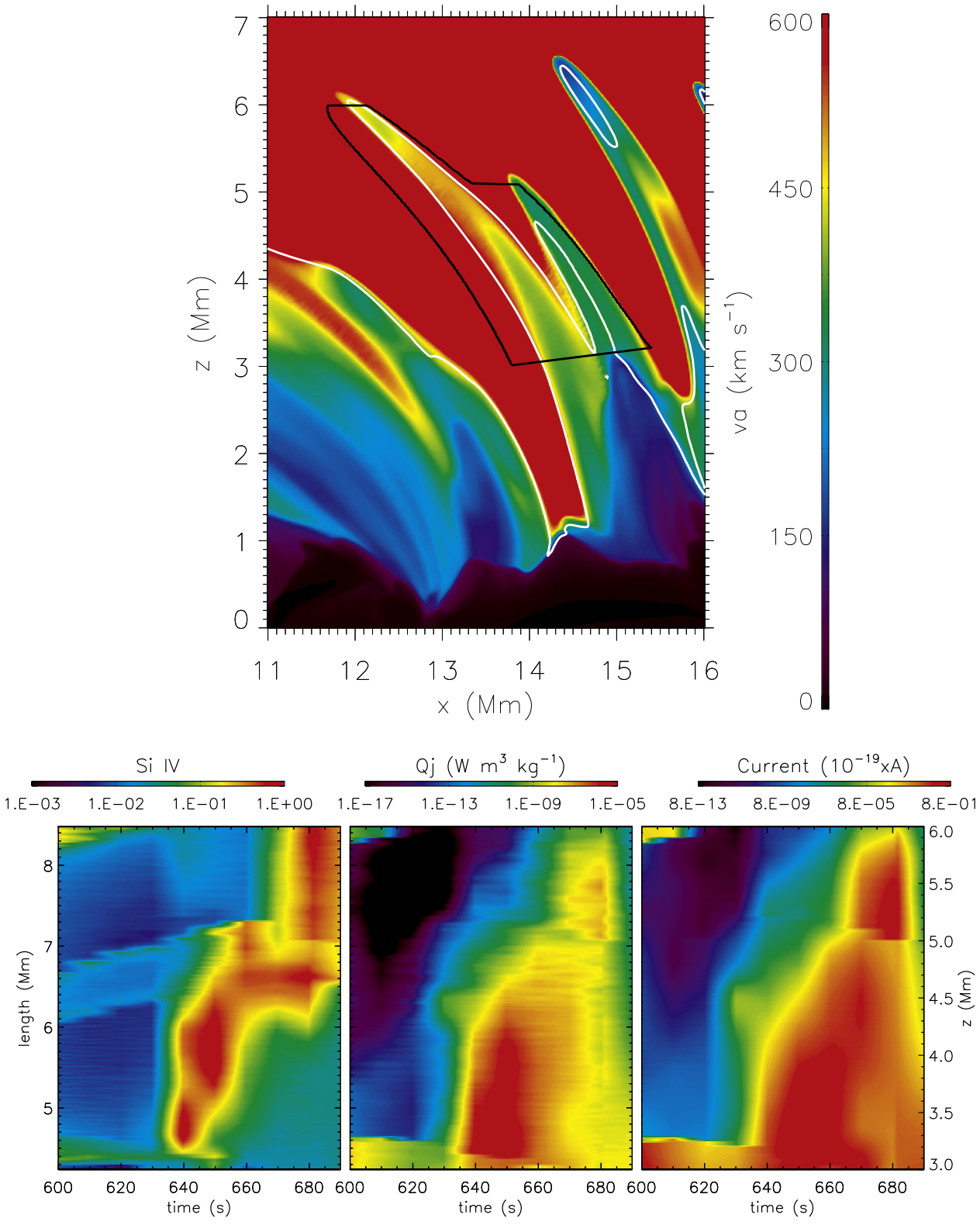

Figure 5. Heating fronts in the simulated spicules propagate with the Alfvén speed. The top shows a map of the Alfvén speed at $t=660 \mathrm{~s}$ (i.e., the same simulation snapshot as in Panels (B) and (F) in Figures 3 and 4) which has values of between 150 and $450 \mathrm{~km} \mathrm{~s}^{-1}$ in the spicule. The white contour shows locations with temperatures of $10^{4} \mathrm{~K}$. The black contour shows the region over which we sum (at each height) to calculate the space-time plots in the bottom row. The bottom row shows, as a function of time, the Si IV $1402 \AA$ emissivity, Joule heating rate and electrical current along the length of the spicule as measured from the photosphere (left vertical axis, for equivalent height, see right vertical axis). The heating front in the right spicule dominates the signal around $t=640 \mathrm{~s}$, while the heating front in the left spicule (at greater heights) occurs around $t=680 \mathrm{~s}$. We see a clear correlation between the rapid Si IV 1402 A increase in both "jets" and Alfvénic propagation of electrical current and associated dissipation.

into the upper chromosphere. The subsequent violent release of magnetic tension drives strong mass flows and heating when the associated electric currents are dissipated. The heating produced by the dissipation of these currents is significant and could potentially play a substantial role in the energy balance of the chromosphere. Further studies will be required to determine whether spicules (or "straws") indeed play a substantial role in heating the chromosphere as tentatively suggested by Cauzzi et al. (2009).

Our results show that there are indeed strong mass motions caused by ejection of spicular material along flux tube-like features. The fast apparent motions in some observables are caused by vigorous heating from the dissipation through ambipolar diffusion of upward propagating electrical currents.
Our results thus indicate that chromospheric spicules or their transition region counterparts do not appear to be caused by random superposition or warping of current sheets along the line of sight (as suggested previously by Judge et al. 2011).

Despite the complex and large variety of physical processes included in this simulation, it suffers from certain limitations which one must address in further studies. This model is limited to only 2.5 spatial dimensions and assumes that Hydrogen and Helium ionization are in statistical equilibrium, whereas in the chromosphere both are far from statistical equilibrium (Leenaarts et al. 2007; Golding et al. 2014). In addition, the generalized Ohm's law is based on limiting assumptions which may break down in the dynamic spicular environment: a full multi-fluid approach will provide further 
insight into the role of ion-neutral interactions in spicules. Finally, while the numerical resolution is high enough to properly treat the ambipolar resistivity, the lack of 3D treatment and resolution means that we cannot exclude the possibility that the actual dissipation mechanism driving the heating fronts is intimately connected to dissipation of Alfvén waves, e.g., through the Kelvin-Helmholtz instability (Antolin et al. 2015).

IRIS is a NASA small explorer mission developed and operated by LMSAL with mission operations executed at NASA Ames Research center and major contributions to downlink communications funded by ESA and the Norwegian Space Centre. We gratefully acknowledge support by NASA grants NNX11AN98G, NNX16AG90G, and NASA contracts NNM07AA01C (Hinode) and NNG09FA40C (IRIS). The simulations were run on clusters from the Notur project, and the Pleiades cluster through the computing project s1061 from the High End Computing (HEC) division of NASA. Snapshots from this numerical simulation (en096014_gol) are publicly available as part of the IRIS modeling archive. Details can be found at http://iris.lmsal.com/modeling.html. To analyze the data, we used IDL.

\section{ORCID iDs}

Bart De Pontieu (1) https://orcid.org/0000-0002-8370-952X Juan Martínez-Sykora (i) https://orcid.org/0000-00020333-5717

Georgios Chintzoglou (1) https://orcid.org/0000-00021253-8882

\section{References}

Antolin, P., Okamoto, T. J., De Pontieu, B., et al. 2015, ApJ, 809, 72 Athay, R. G., \& Holzer, T. E. 1982, ApJ, 255, 743

Beckers, J. M. 1968, SoPh, 3, 367

Carlsson, M., \& Leenaarts, J. 2012, A\&A, 539, A39
Cauzzi, G., Reardon, K., Rutten, R. J., Tritschler, A., \& Uitenbroek, H. 2009, A\&A, 503, 577

De Pontieu, B., Hansteen, V. H., Rouppe van der Voort, L., van Noort, M., \& Carlsson, M. 2007a, ApJ, 655, 624

De Pontieu, B., McIntosh, S., Hansteen, V. H., et al. 2007b, PASJ, 59, 655

De Pontieu, B., McIntosh, S. W., Carlsson, M., et al. 2011, Sci, 331, 55

De Pontieu, B., Rouppe van der Voort, L., McIntosh, S. W., et al. 2014a, Sci, 346, 1255732

De Pontieu, B., Title, A. M., Lemen, J. R., et al. 2014b, SoPh, 289, 2733

Golding, T. P., Carlsson, M., \& Leenaarts, J. 2014, ApJ, 784, 30

Gudiksen, B. V., Carlsson, M., Hansteen, V. H., et al. 2011, A\&A, 531, A154

Hansteen, V. H., De Pontieu, B., Rouppe van der Voort, L., van Noort, M., \& Carlsson, M. 2006, ApJL, 647, L73

Hansteen, V. H., Hara, H., De Pontieu, B., \& Carlsson, M. 2010, ApJ, 718,1070

Hayek, W., Asplund, M., Carlsson, M., et al. 2010, A\&A, 517, A49

Judge, P. G., Tritschler, A., \& Chye Low, B. 2011, ApJL, 730, L4

Kubo, M., Katsukawa, Y., Suematsu, Y., et al. 2016, ApJ, 832, 141

Leenaarts, J., Carlsson, M., Hansteen, V., \& Rutten, R. J. 2007, A\&A, 473,625

Martínez-Sykora, J., De Pontieu, B., \& Hansteen, V. 2012, ApJ, 753, 161

Martínez-Sykora, J., De Pontieu, B., Hansteen, V. H., et al. 2017a, Sci, 356, 1269

Martínez-Sykora, J., De Pontieu, B., Hansteen, V. H., \& Carlsson, M. 2017b, ApJ, 847, 36

Martínez-Sykora, J., Hansteen, V., De Pontieu, B., \& Carlsson, M. 2009, ApJ, 701, 1569

McIntosh, S. W., de Pontieu, B., Carlsson, M., et al. 2011, Natur, 475, 477

Narang, N., Arbacher, R. T., Tian, H., et al. 2016, SoPh, 291, 1129

Pereira, T. M. D., De Pontieu, B., \& Carlsson, M. 2012, ApJ, 759, 18

Raouafi, N. E., Patsourakos, S., Pariat, E., et al. 2016, SSRv, 201, 1

Rouppe van der Voort, L., De Pontieu, B., Pereira, T. M. D., Carlsson, M., \& Hansteen, V. 2015, ApJL, 799, L3

Rouppe van der Voort, L. H. M., De Pontieu, B., Hansteen, V. H. Carlsson, M., \& van Noort, M. 2007, ApJL, 660, L169

Sekse, D. H., Rouppe van der Voort, L., \& De Pontieu, B. 2012, ApJ, 752, 108

Sekse, D. H., Rouppe van der Voort, L., \& De Pontieu, B. 2013, ApJ, 764, 164

Skogsrud, H., Rouppe van der Voort, L., \& De Pontieu, B. 2016, ApJ, 817 124

Skogsrud, H., Rouppe van der Voort, L., De Pontieu, B., \& Pereira, T. M. D. 2015, ApJ, 806, 170

Sterling, A. C. 2000, SoPh, 196, 79

Tian, H., DeLuca, E. E., Cranmer, S. R., et al. 2014, Sci, 346, 1255711

Tsiropoula, G., Tziotziou, K., Kontogiannis, I., et al. 2012, SSRv, 169, 181 